\title{
Miniaturizing transmon qubits using van der Waals materials
}

\author{
Abhinandan Antony \\ Columbia University https://orcid.org/0000-0002-1931-884X
}

\section{Martin Gustafsson}

Raytheon BBN Technologies

\section{Guilhem Ribeill}

Raytheon BBN Technologies

\section{Matthew Ware}

Raytheon BBN Technologies

\section{Anjaly Rajendran}

Columbia University

\section{Luke Govia}

Raytheon BBN Technologies

\section{Thomas Ohki}

Raytheon BBN Technologies

\section{Takashi Taniguchi}

National Institute for Materials Science, Tsukuba, Ibaraki https://orcid.org/0000-0002-1467-3105

\section{Kenji Watanabe}

National Institute for Materials Science https://orcid.org/0000-0003-3701-8119

\section{James Hone}

Columbia University https://orcid.org/0000-0002-8084-3301

Kin Chung Fong ( $\nabla$ fongkc@gmail.com )

Raytheon BBN Technologies https://orcid.org/0000-0002-6558-1083

\section{Article}

Keywords: quantum computers, quibits, van der Waals materials

Posted Date: September 28th, 2021

DOl: https://doi.org/10.21203/rs.3.rs-923599/v1

License: (9) (1) This work is licensed under a Creative Commons Attribution 4.0 International License. 
Version of Record: A version of this preprint was published at Nano Letters on November 18th, 2021. See the published version at https://doi.org/10.1021/acs.nanolett.1c04160. 


\title{
Miniaturizing transmon qubits using van der Waals materials
}

\author{
Abhinandan Antony, ${ }^{1}$ Martin V. Gustafsson, ${ }^{2}$ Guilhem J. Ribeill, ${ }^{2}$ Matthew Ware,${ }^{2}$ \\ Anjaly Rajendran, ${ }^{3}$ Luke C. G. Govia,${ }^{2}$ Thomas A. Ohki, ${ }^{2}$ Takashi Taniguchi, ${ }^{4}$ \\ Kenji Watanabe, ${ }^{5}$ James Hone, ${ }^{1}$ Kin Chung Fong ${ }^{2 *}$ \\ ${ }^{1}$ Department of Mechanical Engineering \\ Columbia University, New York, NY 10027, USA \\ ${ }^{2}$ Raytheon BBN Technologies, Quantum Engineering and Computing Group \\ Cambridge, Massachusetts 02138, USA \\ ${ }^{3}$ Department of Electrical Engineering \\ Columbia University, New York, NY 10027, USA \\ ${ }^{4}$ International Center for Materials Nanoarchitectonics, \\ National Institute for Materials Science, Tsukuba, Japan \\ ${ }^{5}$ Research Center for Functional Materials, \\ National Institute for Materials Science, Tsukuba, Japan
}

*To whom correspondence should be addressed; E-mail: kc.fong@rtx.com. 
Quantum computers can potentially achieve an exponential speedup versus classical computers on certain computational tasks, recently demonstrated in systems of superconducting qubits. However, the capacitor electrodes that comprise these qubits must be large in order to avoid lossy dielectrics. This tactic hinders scaling by increasing parasitic coupling among circuit components, degrading individual qubit addressability, and limiting the spatial density of qubits. Here, we take advantage of the unique properties of van der Waals (vdW) materials to reduce the qubit area by $>1000$ times while preserving the required capacitance without increasing substantial loss. Our qubits combine conventional aluminum-based Josephson junctions with parallel-plate capacitors composed of crystalline layers of superconducting niobium diselenide and insulating hexagonal-boron nitride. We measure a vdW transmon $T_{1}$ relaxation time of $1.06 \mu \mathrm{s}$, which demonstrates a path to achieve high-qubitdensity quantum processors with long coherence times, and the broad utility of layered heterostructures in low-loss, high-coherence quantum devices.

Superconducting qubits form an attractive platform to build scalable quantum computers, as recently demonstrated in quantum processors consisting of large arrays of qubits (1). Achieving fault-tolerant quantum computation would provide a transformative technology in quantum simulation, chemistry, and optimization $(2,3)$. However, the industry-standard transmon qubit consists of Josephson junctions (JJ) shunted by capacitors with a large footprint of $\sim 10^{5} \mu \mathrm{m}^{2}$. In order to acquire a sufficient capacitance, these capacitors are made of large planar electrodes without dielectric materials that can host two-level systems (TLSs), which are a major source of decoherence (4). While brute-force scaling using conventional transmons remains possible (5), there is growing interest in alternative approaches that could lay the foundation of next-generation quantum processors. Approaches such as protected qubits can decouple from 
environmental noise (6), but improving the constituent materials can dramatically impact qubit technology by reducing the footprint and enhancing qubit coherence. As such, new material platforms for qubits are now actively explored (7-13).

Van der Waals (vdW) heterostructures are a promising material platform for quantum devices. Cleaved from pure bulk crystals, the surfaces of these heterostructures are pristine and atomically flat. They can be assembled into stacks with their layers held together by weak bonds without straining the crystals (14). This architecture allows for diverse properties of various vdW materials, such as gate-tunability $(15,16)$, high kinetic inductance (17), and giant thermal response to microwave photons $(18,19)$, which can be exploited for potential quantum applications (20). Here, we take advantage of the high-quality vdW heterostructures to fabricate compact vdW parallel-plate capacitors (PPC) using superconducting niobium diselenide $\left(\mathrm{NbSe}_{2}\right)$ and insulating hexagonal boron nitride $(\mathrm{hBN})$. We observe quantum coherence of a transmon qubit that uses a vdW PPC as the shunt capacitor. Our result goes against the current trend of requiring large-footprint shunt capacitors in transmons (5), and demonstrates the potential of using crystalline materials to improve qubit coherence.

Fig. 1a shows schematically the fabrication process of the vdW PPC (13) (details in Supplementary Information). It is different from the conventional process for making high-quality $\mathrm{vdW}$ heterostructures (14) because qubits operate in the single-photon regime, where any dissipation or loss channel introduced by the materials or fabrication process can destroy quantum information. Therefore, we assemble each heterostructure within an inert atmosphere to avoid oxidation and contamination of the interfaces. Then, the vdW heterostructure is placed onto the qubit substrate with pre-patterned niobium resonators. To make a vdW transmon, we connect the vdW PPC in parallel with two $\mathrm{Al}-\mathrm{AlO}_{x}-\mathrm{Al} \mathrm{JJs}$ that form a superconducting quantum interference device (SQUID) loop for tuning of the qubit frequency with a magnetic flux generated by a bias current through an off-chip coil. A conventional qubit formed using a planar $\mathrm{Nb}$ ca- 
pacitor and a single $\mathrm{JJ}$ is created at the same time for comparison. Fig. $1 \mathrm{~b}$ schematically shows these elements, with the transmons capacitively coupled to the readout resonator (red) and drive line (green). Fig. 1c and 1d show optical micrographs of the assembled vdW PPC (109 $\mu \mathrm{m}^{2}$ in area and $35 \mathrm{~nm}$ in thickness) and the vdW transmon, respectively. The optical image in Fig. 1e shows the dramatic size reduction enabled by the vdW PPC, which is about 1400 times smaller than the conventional planar shunt capacitor. For this study, we fabricated and measured two $\mathrm{vdW}$ transmons. We fully characterize one of them and present the data in the main text.

We perform spectroscopy experiments to characterize the coupling of the vdW qubit to its readout cavity. With high probing power, the cavity is unperturbed by the qubit and resonates at $6.9073 \mathrm{GHz}$ with a linewidth of $290 \mathrm{kHz}$ (Fig. 2a). Measuring under low power, we find the strong coupling with the qubit dispersively shifts the cavity frequency by $1.00 \mathrm{MHz}(21)$. Fig. $2 b$ shows how the flux-bias current modulates the cavity frequency. We perform qubit spectroscopy by applying a control signal at various frequencies to the qubit while probing the cavity nearly on resonance. Fig. $2 \mathrm{c}$ shows the transition frequency, $\omega_{01} / 2 \pi$, between the ground, $|0\rangle$, and excited, $|1\rangle$, states of the qubit at $5.2815 \mathrm{GHz}$. Using the two-photon transition frequency between the $|0\rangle$ and $|2\rangle$ states, i.e. $\omega_{02} / 2 \pi=5.216 \mathrm{GHz}$, we find the qubit anharmonicity, $\alpha / 2 \pi,=-131 \mathrm{MHz} . \alpha$ agrees with the charging energy of the qubit, $E_{C}\left(-\alpha=E_{C} / h\right.$ for a transmon with $h$ being the Planck constant), calculated using the PPC formula with an hBN dielectric constant of 4.4. Fig. $2 d$ shows $\omega_{01} / 2 \pi$ versus flux-bias current. It has a maximum at $\approx 0.2 \mathrm{~mA}$, which is consistent with the flux dependence of the readout resonator in Fig. $2 \mathrm{a}$ and represents the sweet spot where the transmon is the least susceptible to flux noise. In $5-\mu \mathrm{A}$ steps of flux-bias current, we observe no anticrossing in the qubit spectroscopy due to the coupling of the vdW transmon with coherent TLSs. From the dispersive shift and detuning from the readout cavity, $\Delta$, of $1.6258 \mathrm{GHz}$ at the sweet spot, we find the qubit-resonator coupling, $g / 2 \pi,=40.3 \mathrm{MHz}$. The Josephson energy, $E_{J} / h$, calculated from $\omega_{01}$, is $28.0 \mathrm{GHz}$. Our qubit 
is in the transmon regime with $E_{J} / E_{C} \simeq 214$.

We demonstrate the quantum coherence of the vdW transmon by performing Rabi experiments (see Fig. 3a). By driving the qubit with a resonant pulse for a time, $t_{\text {Rabi }}$, the vdW transmon Rabi oscillates coherently on the Bloch sphere between the ground, $|0\rangle$, and excited, $|1\rangle$, states before being measured using the dispersive shift of the resonator (21). The oscillation frequency, $\omega_{\mathrm{Rabi}} / 2 \pi$ is set by the drive strength, $\Omega$, and drive detuning from the qubit frequency, $\delta \omega_{d}$, as $\omega_{\text {Rabi }}=\sqrt{\Omega^{2}+\delta \omega_{d}^{2}}$. When performed at different excitation frequencies and a high pulse power, we obtain the data as shown in Fig. 3b. We quantify the qubit lifetime by measuring the $T_{1}$ energy relaxation time at the sweet spot (Fig. 3c). After applying an 80-ns-long $\pi$-pulse to excite the qubit from $|0\rangle$ to $|1\rangle$ and waiting for a time delay, $t_{\text {delay }}$, the qubit state is read out. We repeatedly measure the exponential decay of excited state probability, $P_{|1\rangle}$, to quantify the mean and fluctuations of $T_{1}$ over a period of 8 hours. The histogram (see inset) shows $T_{1}$ of $1.06 \mu \mathrm{s} \pm 0.09 \mu \mathrm{s}$ (standard deviation). Next, we quantify the qubit coherence by measuring the dephasing time $T_{2}^{*}$ during a Ramsey experiment. Fig. $3 \mathrm{~d}$ shows the qubit readout signal after the Ramsey pulse sequence of $\pi / 2-t_{\text {delay }}-\pi / 2$. The oscillation is due to $\delta \omega_{d}$. We fit the data to a decay oscillation and obtain $T_{2}^{*}$ of $1.67 \pm 0.20 \mu$ s. Fig. 3e shows Ramsey oscillations at different qubit drive frequencies. Finally, we probe for evidence of excessive low frequency noise that may dephase the vdW transmon using a Hahn echo pulse sequence, which contains an additional $\pi$-pulse in the middle of the Ramsey sequence. Fig. 3f shows the qubit readout signal after the Hahn echo sequence. We obtain the echo dephasing time, $T_{2 E}$, of $1.72 \pm 0.27 \mu$ s, that is nearly energy-relaxation limited, i.e. $T_{2 E} \approx 2 T_{1}$.

We can compare the performance of our vdW transmon with the conventional one fabricated on the same substrate. The $T_{1}$ and $T_{2}^{*}$ of the conventional transmon are $11.5 \pm 0.4 \mu \mathrm{s}$ and $10.5 \pm 0.6 \mu \mathrm{s}$, respectively. Since both transmons are subjected to the same fabrication process and measurement setup environment, this result suggests that the JJs are not the limiting factor 
of the quantum coherence of our vdW transmon. On the other hand, based on finite-element simulations, only $87 \%$ of the capacitive energy of our vdW transmon design is confined internally to the PPC. This could result in degraded qubit performance through a finite qubit coupling with residue, external to the PPC, from the vdW stacking process or Al lift-off. If we assume the energy relaxation is entirely caused by the capacitive loss and use the equation, $1 / T_{1}=\omega_{01} \tan \delta$, we can estimate the upper bound of the loss tangent, $\tan \delta$, of the vdW PPC to be $2.83 \times 10^{-5}$. This value is not the intrinsic $\tan \delta$ of the $\mathrm{hBN}$, but is approaching the measured values from PPCs using silicon as the dielectric $\left(\approx 10^{-6}\right)(22,23)$. To extend qubit $T_{1}$ relaxation, we will need to improve the fabrication process and further concentrate the electric field within the pristine interior of the vdW PPC.

Recently, experiments have taken a similar direction using the intrinsic JJ PPC to make the merged-element transmon (MET) $(12,24)$. While both the MET and vdW transmon accomplish miniaturization, the METs demonstrated so far use amorphous insulators which are known to harbor TLSs. As the vdW heterostructure can also function as a high-quality $\mathrm{JJ}(8,25,26)$, one can envision a future vdW-MET consisting of a crystalline vdW material as the tunnel barrier. Moreover, the thickness of the vdW insulator is defined by discrete atomic layers. This highlyuniform, crystalline tunnel barrier can potentially allow for a reduced TLS defect density and precise control of qubit frequencies, which can address the frequency-crowding problem (27). In the future, we plan to extend our result to further miniaturize quantum processors by reducing footprints of circuit components using, e.g. compact lumped-element resonators.

During the preparation of this manuscript, we became aware of a complementary work (28). 


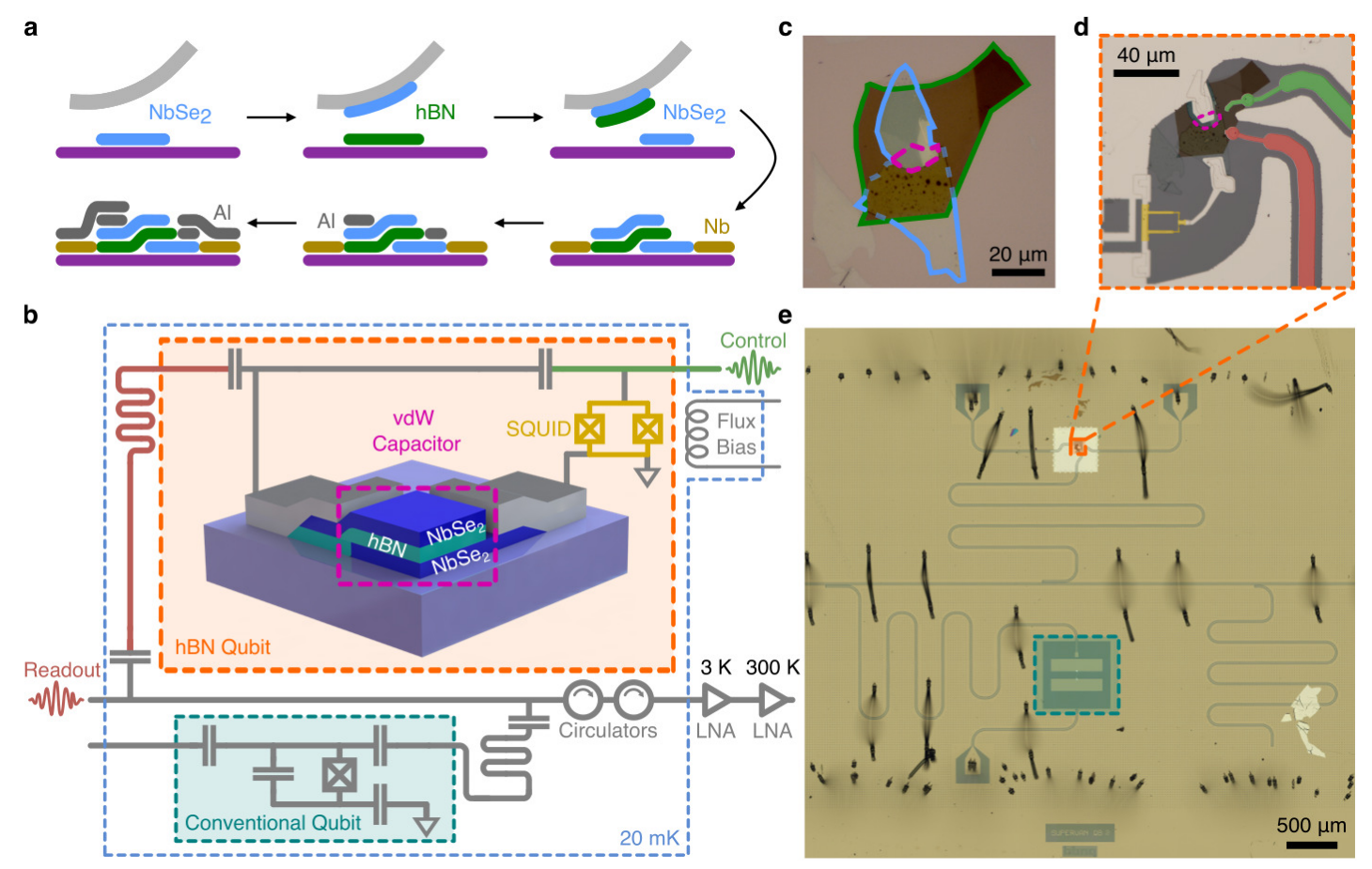

Figure 1: Fabrication of vdW transmon and measurement schematics. (a) Fabrication procedures for the vdW parallel-plate capacitor (PPC). In clockwise direction: dry polymer-based stacking of vdW heterostructure by sequential pick-up of $\mathrm{NbSe}_{2}, \mathrm{hBN}$, and $\mathrm{NbSe}_{2}$ flakes. Transfer of the heterostructure to a void within the pre-patterned $\mathrm{Nb}$ layer on a silicon substrate, deposition of $\mathrm{Al}$ contacts, and deposition of JJs and interconnects. (b) Schematic circuit diagram showing our frequency-tunable qubit (orange box) and fixed-frequency conventional qubit (teal box) as a control. Both qubits are coupled to coplanar-waveguide resonators for readout through a common bus line with low noise amplifiers (LNA) after circulators. (c) Optical micrograph of the $\mathrm{NbSe}_{2}-\mathrm{hBN}-\mathrm{NbSe}_{2}$ heterostructure used to make the PPC (pink dashed line) with an area of $109 \mu \mathrm{m}^{2}$ and $\mathrm{hBN}$ thickness of $35 \mathrm{~nm}$. (d) Optical micrograph of the vdW transmon formed by the SQUID loop (yellow) shunted by the vdW capacitor with capacitive coupling to the readout resonator (red) and control line (green). (e) Optical micrograph of both qubits in the same field of view, illustrating the miniaturization of transmon qubit by the vdW capacitor. 

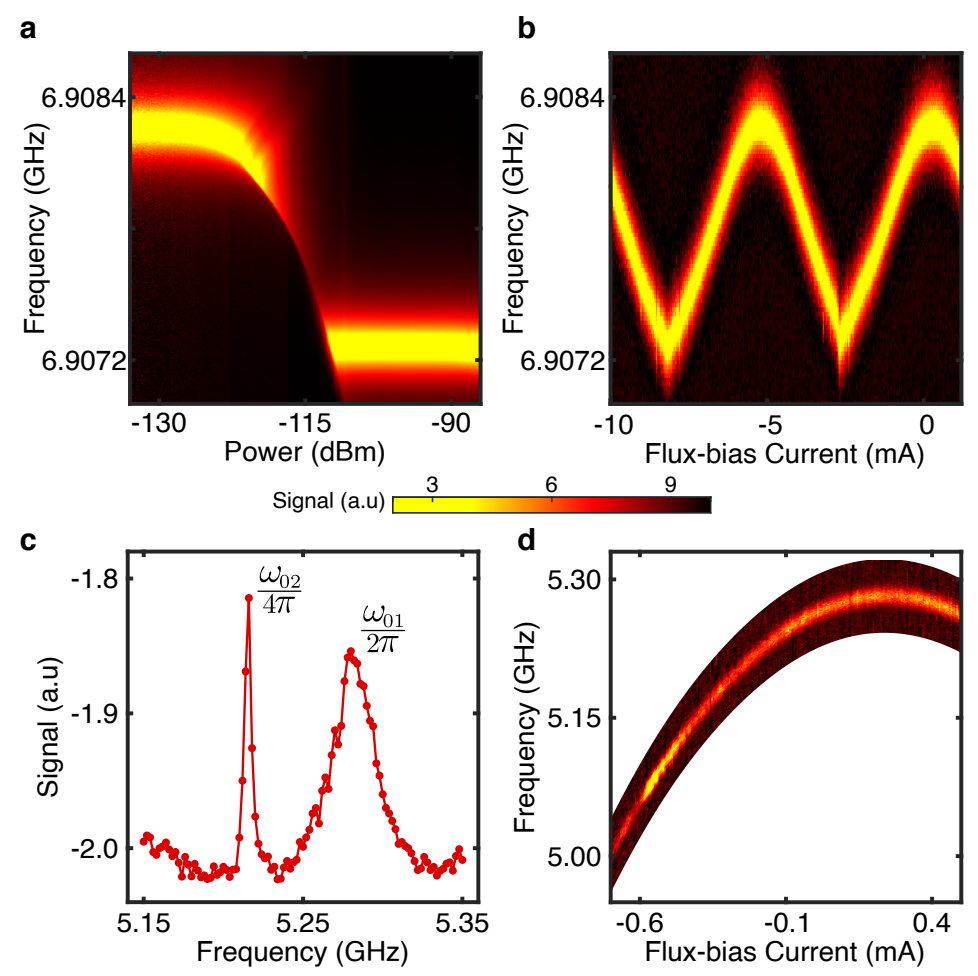

Figure 2: Spectroscopy of a vdW transmon (a) Readout-cavity resonance as a function of applied microwave power at the sweet spot of flux bias. (b) Readout-cavity resonance versus flux-bias current. (c) High power spectroscopy shows the $\omega_{01}$ and two-photon $\omega_{02} / 2$ qubit transitions at the sweet spot of flux bias. (d) Qubit frequency, $\omega_{01} / 2 \pi$, as a function of flux tuning. 

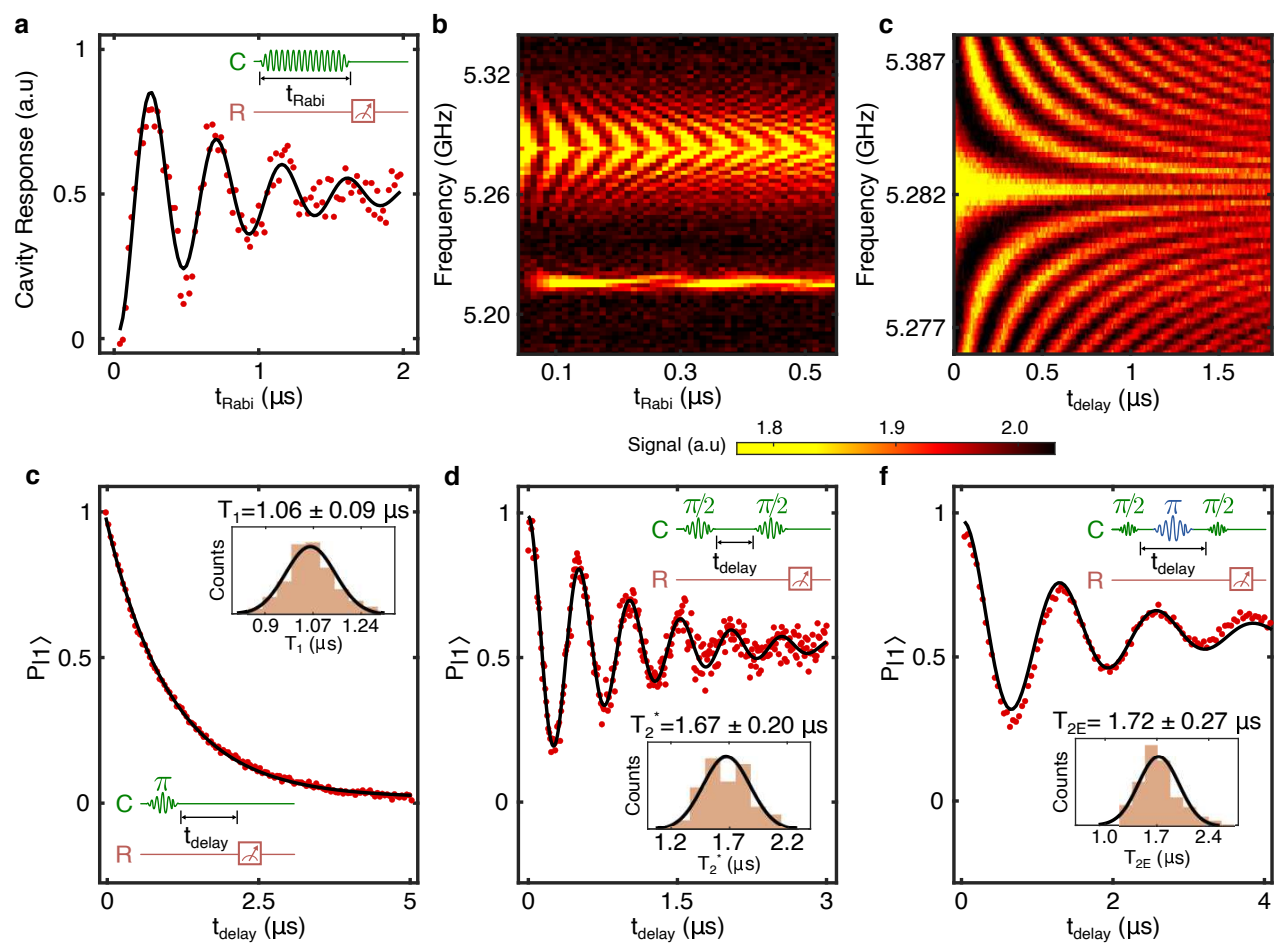

Figure 3: Quantum coherence of the vdW transmon at the sweet spot of flux bias. (a) Rabi oscillation vs. the pulse width, $t_{\text {Rabi }}$ near the qubit frequency. (b) Rabi oscillations at a high power for different excitation frequencies. (c) $T_{1}$ energy relaxation time measurement with the pulse control and measurement sequence (inset). The exponential fit yields the $T_{1}$ relaxation time. The inset histogram shows data from 8 hours of repeated measurements. d) Ramsey experiment shows $T_{2}^{*}$ dephasing time. (e) Ramsey fringes at different drive tone frequencies. (f) Hahn echo experiment to obtain the echo dephasing time, $T_{2 E}$. 


\section{References}

1. Arute, F. et al. Quantum supremacy using a programmable superconducting processor. Nature 574, 505-510 (2019).

2. Cao, Y. et al. Quantum Chemistry in the Age of Quantum Computing. Chem. Rev. 119, 10856 (2019).

3. Berry, D. W., Childs, A. M., Su, Y., Wang, X. \& Wiebe, N. Time-dependent Hamiltonian simulation with $L^{1}$-norm scaling. Quantum 4, 254 (2020).

4. Müller, C., Cole, J. H. \& Lisenfeld, J. Towards understanding two-level-systems in amorphous solids: insights from quantum circuits. Rep. Prog. Phys. 82, 124501 (2019).

5. Gambetta, J. M. et al. Investigating Surface Loss Effects in Superconducting Transmon Qubits. IEEE Trans. Appl. Supercond. 27, 1-5 (2017).

6. Gyenis, A. et al. Experimental Realization of a Protected Superconducting Circuit Derived from the 0- $\pi$ Qubit. PRX Quantum 2, 010339 (2021).

7. Casparis, L. et al. Superconducting gatemon qubit based on a proximitized twodimensional electron gas. Nat. Nanotechnol. 13, 915-919 (2018).

8. Lee, K.-H. et al. Two-Dimensional Material Tunnel Barrier for Josephson Junctions and Superconducting Qubits. Nano Lett. 19, 8287 (2019).

9. Wisbey, D. S. et al. Dielectric Loss of Boron-Based Dielectrics on Niobium Resonators. J. Low Temp. Phys. 195, 474-486 (2019).

10. McRae, C. R. H. et al. Cryogenic microwave loss in epitaxial Al/GaAs/Al trilayers for superconducting circuits. J. Appl. Phys. 129, 025109 (2021). 
11. Place, A. P. M. et al. New material platform for superconducting transmon qubits with coherence times exceeding 0.3 milliseconds. Nat. Comm. 12, 1-6 (2021).

12. Mamin, H. J. et al. Merged-Element Transmons: Design and Qubit Performance. Phys. Rev. Appl. 16, 024023 (2021).

13. Antony, A. et al. Making high-quality quantum microwave devices with van der Waals superconductors. Preprint at https://arxiv.org/abs/2107.09147 (2021).

14. Wang, L. et al. One-Dimensional Electrical Contact to a Two-Dimensional Material. Science 342, 614-617 (2013).

15. Schmidt, F. E., Jenkins, M. D., Watanabe, K., Taniguchi, T. \& Steele, G. A. A ballistic graphene superconducting microwave circuit. Nat. Comm. 9, 4069 (2018).

16. Wang, J. I.-J. et al. Coherent control of a hybrid superconducting circuit made with graphene-based van der Waals heterostructures. Nat. Nanotechnol. 14, 120-125 (2019).

17. Seifert, P. et al. Magic-Angle Bilayer Graphene Nanocalorimeters: Toward Broadband, Energy-Resolving Single Photon Detection. Nano Lett. 20, 3459 (2020).

18. Lee, G.-H. et al. Graphene-based Josephson junction microwave bolometer. Nature 586, 42-46 (2020).

19. Kokkoniemi, R. et al. Bolometer operating at the threshold for circuit quantum electrodynamics. Nature 586, 47-51 (2020).

20. Liu, X. \& Hersam, M. C. 2D materials for quantum information science. Nat. Rev. Mater. 4, 669-684 (2019). 
21. Blais, A., Grimsmo, A. L., Girvin, S. M. \& Wallraff, A. Circuit quantum electrodynamics. Rev. Mod. Phys. 93, 025005 (2021).

22. Patel, U. et al. Coherent Josephson phase qubit with a single crystal silicon capacitor. Appl. Phys. Lett. 102, 012602 (2013).

23. Sandberg, M. et al. Radiation-suppressed superconducting quantum bit in a planar geometry. Appl. Phys. Lett. 102, 072601 (2013).

24. Zhao, R. et al. Merged-Element Transmon. Phys. Rev. Appl. 14, 064006 (2020).

25. Island, J. O., Steele, G. A., van der Zant, H. S. J. \& Castellanos-Gomez, A. Thickness dependent interlayer transport in vertical MoS2 Josephson junctions. 2D Materials 3, 031002 (2016).

26. Dvir, T. et al. Spectroscopy of bulk and few-layer superconducting NbSe 2 with van der Waals tunnel junctions. Nat. Comm. 9, 1-6 (2018).

27. Brink, M., Chow, J. M., Hertzberg, J., Magesan, E. \& Rosenblatt, S. Device challenges for near term superconducting quantum processors: frequency collisionsDevice challenges for near term superconducting quantum processors: frequency collisions. IEEE International Electron Devices Meeting IEDM 6.1.1 (2018).

28. Wang, J. I. J. et al. Hexagonal Boron Nitride (hBN) as a Low-loss Dielectric for Superconducting Quantum Circuits and Qubits. Preprint at https://arxiv.org/abs/2109.00015 (2021).

\section{Acknowledgments}

We thank L. Ranzani for useful discussions. Most of this work was supported by Army Research Office under Contract Number W911NF-18-C-0044. Development of heterostructure assembly 
techniques at Columbia was supported by the NSF MRSEC program (DMR-2011736). A.A. thanks the supplemental support from QISE-NET under NSF DMR-1747426. K.W. and T.T. acknowledge support from the Elemental Strategy Initiative conducted by the MEXT, Japan (Grant Number JPMXP0112101001) and JSPS KAKENHI (Grant Numbers JP19H05790 and JP20H00354). 


\section{Supplementary information}

\section{List of supplementary materials:}

- Methods

- Figures S4-S5

- Table S1

\section{Methods}

Flakes of hexagonal-boron nitride $(\mathrm{hBN})$ and niobium diselenide $\left(\mathrm{NbSe}_{2}\right)$ of thickness about 35 and $20 \mathrm{~nm}$, respectively, were exfoliated onto silicon substrates with $285 \mathrm{~nm} \mathrm{SiO}{ }_{2}$ epilayers. The thickness of each hBN flake was measured using atomic force microscopy prior to stacking. Because the $\mathrm{NbSe}_{2}$ is highly air sensitive, $\mathrm{NbSe}_{2}$ exfoliation and assembly of the $\mathrm{NbSe}_{2} / \mathrm{hBN} / \mathrm{NbSe}_{2}$ heterostructure (Fig. 1A) was performed inside the inert atmosphere of a nitrogen filled glovebox, using a polypropylene carbonate/Polydimethylsiloxane based dry pick-up and transfer process (14). Since the loss in the polypropylene carbonate is one of the major causes of qubit relaxation, special care was taken to keep the $\mathrm{NbSe}_{2}-\mathrm{hBN}-\mathrm{NbSe}_{2}$ interfaces clean. The heterostructure was then transferred to a $400 \mu \mathrm{m} \times 400 \mu \mathrm{m}$ sized bare silicon landing site, on a high resistivity Si substrate coated with $200 \mathrm{~nm}$ thick niobium (Nb). Coplanar transmission lines for control and readout resonators, as well as coplanar capacitor pads for a secondary benchmark/control qubit were patterned into the $\mathrm{Nb}$ layer prior to the incorporation of vdW materials. E-beam lithography was used to define Ti/Al contacts to the two $\mathrm{NbSe}_{2}$ flakes. In situ ion milling to remove the oxide that forms on the exposed $\mathrm{NbSe}_{2}$ surfaces prior to deposition was crucial to achieving ultralow-loss contacts (13). $\mathrm{Al}-\mathrm{AlO}_{x}-\mathrm{Al} \mathrm{JJs}$ were fabricated using standard e-beam lithography and double-angle evaporation. Further steps of e-beam lithography and high quality $\mathrm{Al}$ deposition were performed to connect the $\mathrm{Nb}$ resonators and 
ground plane to the contacts and to create superconducting interconnects. 

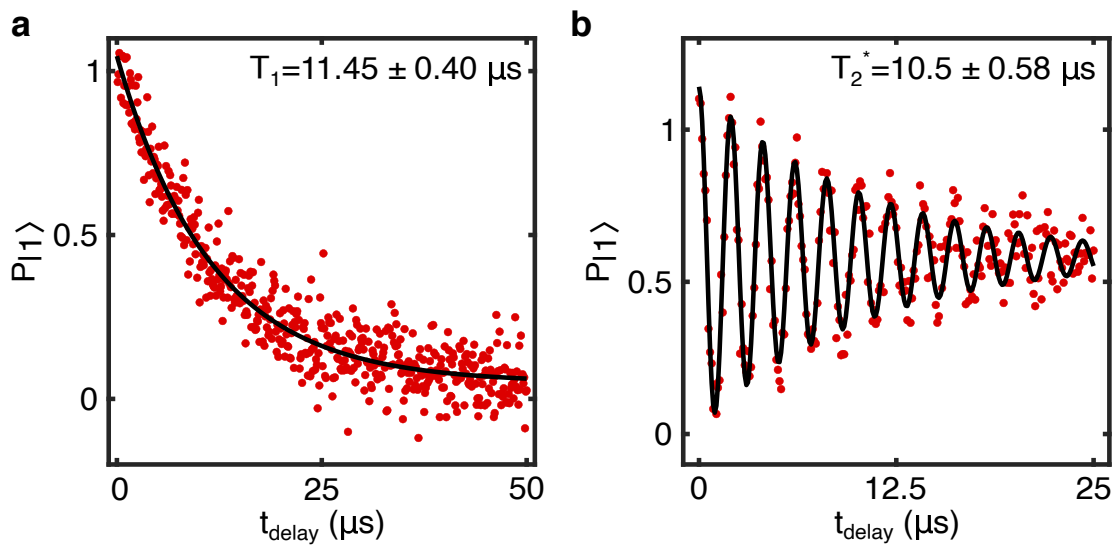

Figure S4: Quantum coherence of the Conventional transmon (a) $T_{1}$ energy relaxation time measurement. (b) Ramsey experiment shows $T_{2}^{*}$ dephasing time.
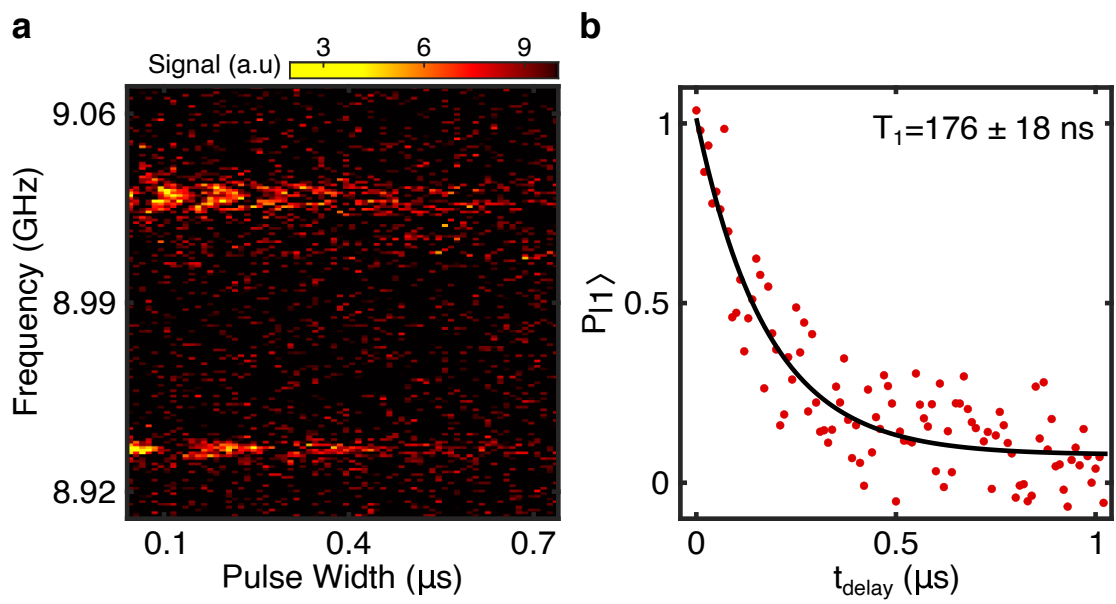

Figure S5: Quantum coherence of the second vdW transmon device. (a) Rabi oscillations at a high power at different excitation frequencies. (b) $T_{1}$ energy relaxation time measurement. 


\begin{tabular}{lc}
\hline Physical quantity & Value \\
\hline Readout-cavity frequency, $\omega_{c} / 2 \pi$ & $6.9083 \mathrm{GHz}$ \\
vdW transmon frequency, $\omega_{01} / 2 \pi$ & $5.2815 \mathrm{GHz}$ \\
Anharmonicity, $\alpha / 2 \pi$ & $-131 \mathrm{MHz}$ \\
Coupling strength, $g / 2 \pi$ & $40.3 \mathrm{MHz}$ \\
Detuning, $\Delta / 2 \pi$ & $1.6258 \mathrm{GHz}$ \\
Josephson energy, $E_{J} / h$ & $28.0 \mathrm{GHz}$ \\
Charging energy, $E_{C} / h$ & $131 \mathrm{MHz}$ \\
$E_{J} / E_{C}$ & 214 \\
$\mathrm{JJ}$ normal resistance, $R_{n}$ & $11.2 \mathrm{k} \Omega$ \\
$\mathrm{JJ}$ critical current, $I_{c}$ & $11.99 \mu \mathrm{A}$ \\
$E_{J}^{\prime}=\Phi_{0} I_{c} / 2 \pi$ & $27.4 \mathrm{GHz}$ \\
PPC area & $109 \mu \mathrm{m}^{2}$ \\
PPC (hBN) thickness & $35 \mathrm{~nm}$ \\
PPC capacitance & $110 \mathrm{fF}$ \\
\hline
\end{tabular}

Table S1: List of vdW parameters. Values from device S111 that is presented in the main text. $R_{n}$ is measured at room temperature from a $\mathrm{JJ}$ fabricated on the same qubit substrate. $I_{c}$ is inferred from Ambegaokar-Baratoff relation using $R_{n}$. $E_{J}$ is calculated using $\omega_{01}$ and $\alpha$ whereas $E_{J}^{\prime}=\Phi_{0} I_{c} / 2 \pi$ is the Josephson energy calculated using $I_{c}$ with $\Phi_{0}$ being the magnetic flux quanta. All quantities, if dependent on the flux-bias, are taken at the sweet spot. 


\section{Supplementary Files}

This is a list of supplementary files associated with this preprint. Click to download.

- SuperVanQubitV2Supp.pdf 